\title{
Modular interface and experimental setup for in-vacuum operation of microfluidic devices
}

D. Maillard, A. De Pastina, T. Larsen, L.G. Villanueva

This article may be downloaded for personal use only. Any other use requires prior permission of the author and AIP Publishing. This article appeared in D. Maillard et al., Review of Scientific Instruments 90, 045006 (2019) and may be found at https://doi.org/10.1063/1.5088946 


\section{Modular interface and experimental setup for in-vacuum operation of microfluidic devices}

Cite as: Rev. Sci. Instrum. 90, 045006 (2019); https://doi.org/10.1063/1.5088946

Submitted: 15 January 2019 . Accepted: 09 April 2019. Published Online: 29 April 2019

Damien Maillard, Annalisa De Pastina, Tom Larsen, and (D) Luis Guillermo Villanueva

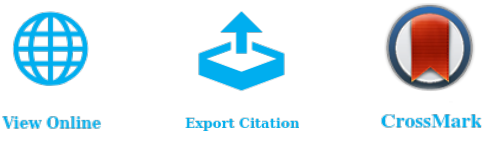

\section{ARTICLES YOU MAY BE INTERESTED IN}

Engineered acoustic mismatch for anchor loss control in contour mode resonators

Applied Physics Letters 114, 103502 (2019); https://doi.org/10.1063/1.5086156

Effective quality factor tuning mechanisms in micromechanical resonators

Applied Physics Reviews 5, 041307 (2018); https://doi.org/10.1063/1.5027850

Effect of AIN seed layer on crystallographic characterization of piezoelectric AIN

Journal of Vacuum Science \& Technology A 37, 021504 (2019); https://

doi.org/10.1116/1.5082888

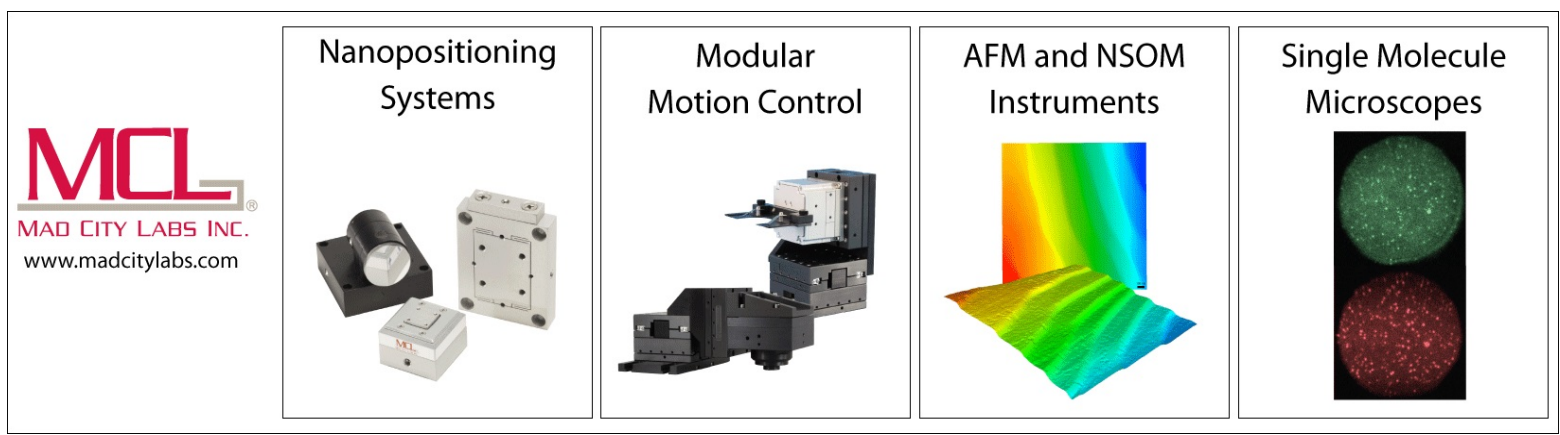

Rev. Sci. Instrum. 90, 045006 (2019); https://doi.org/10.1063/1.5088946 


\title{
Modular interface and experimental setup for in-vacuum operation of microfluidic devices
}

\author{
Cite as: Rev. Sci. Instrum. 90, 045006 (2019); doi: $10.1063 / 1.5088946$ \\ Submitted: 15 January 2019 • Accepted: 9 April 2019 • \\ Published Online: 29 April 2019
}

Damien Maillard, ${ }^{\text {,) }}$ (D) Annalisa De Pastina, (D) Tom Larsen, and Luis Guillermo Villanueva (i)

\begin{abstract}
AFFILIATIONS
Advanced NEMS Laboratory, Institute of Mechanical Engineering, École Polytechnique Fédérale de Lausanne (EPFL), 1015 Lausanne, Switzerland
\end{abstract}

${ }^{\text {a) }}$ Author to whom correspondence should be addressed: damien.maillard@epfl.ch.

\begin{abstract}
We report on the design and operation of a world-to-chip microfluidic interface and experimental setup for fluidic micro- and nanoelectromechanical systems. The central component of the interface is an engineered polyether ether ketone connector that brings fluid samples from a commercial syringe pump to the chip with the help of o-rings. In addition to that, the connector serves as an on-chip vacuum chamber. To confirm the adequate operation of our interface, we use complex microfluidic devices that were previously fabricated, suspended microchannel resonators, and demonstrate a fast exchange between fluids (on the scale of $130 \mathrm{~s}$ from isopropyl alcohol to water), in-vacuum operation of the devices (intrinsic damping regime), and accurate temperature control of the chip at different set points.
\end{abstract}

Published under license by AIP Publishing. https://doi.org/10.1063/1.5088946

\section{INTRODUCTION}

Micro- and nano-electromechanical systems (M/NEMS) are continuously gaining interest in the academic and industrial communities due to the outstanding capabilities they offer to fields as diverse as telecommunications, ${ }^{1}$ biotechnology, ${ }^{2,3}$ or consumer electronics, ${ }^{4}$ among others.

An important issue to address with M/NEMS is the question of the world-to-chip interface, the complexity of which depends closely on the purpose the device serves. For example, MEMS in biological applications require a fluidic network (often made of polydimethyl siloxane, best known under its acronym PDMS) to bring analytes to the detection site. ${ }^{5}$ Mechanical resonators exhibit lower energy losses if they are operated in a vacuum environment and hence show an enhanced quality factor. ${ }^{6}$ Every MEMS with electrical transduction needs appropriate electrical connections to transfer information sensed at the device level to the outside world for monitoring. ${ }^{7}$ Additionally, an accurate control of the temperature is needed for sensors or oscillators. While implementing a single one of these requirements is rather easy, combining many of them in a comprehensive setup requires a more complex engineering approach.

In this paper, we present a reversible, modular, o-ring-based experimental platform for the operation of electrically transduced fluidic M/NEMS operated in a vacuum environment. We start by listing the different components of the interface, the central part of which is a custom-made polyether ether ketone (PEEK) connector. We then elaborate on the three characteristics of the interface that we highlight as the most important, namely a fast exchange between fluids, in-vacuum operation of the devices, and accurate temperature control.

All the modules developed in this interface are successfully tested with previously fabricated piezoelectrically transduced suspended microchannel resonators (SMRs). ${ }^{8,9}$ SMRs are resonant beams with embedded microfluidic channels and have been of great interest in the field of biological applications to measure the density and viscosity of picoliter fluid samples, ${ }^{10}$ to determine masses, densities, and sizes of populations of nanoparticles and bacteria, ${ }^{11,12}$ and to monitor single-cell growth rates. ${ }^{13}$ Due to their complex characteristics, SMRs are demanding devices in terms of interface and setup. For this reason, they firmly validate the operation of our platform.

\section{WORLD-TO-CHIP INTERFACE}

The complexity of SMRs set the diverse, intricate requirements demanded for the optimal operation of their world-to-chip interface:

(i) The platform must ensure efficient fluid delivery to the SMRs. (ii) Operation of the devices in a vacuum environment is a key feature that allows us to reduce damping induced by the air 
surrounding the resonators, thus reaching higher quality factors. (iii) The resonators need to be visually accessible for monitoring purposes, i.e., checking for air bubbles or clogs in the fluidic channels and inspecting the cells and allow optical-based detection of the SMRs motion with an external source (in our case a Laser Doppler Vibrometer; Polytec, OFV-551). (iv) Temperature of the devices must be kept as stable as possible to limit undesirable fluctuations in resonance frequency. (v) The transduction electrode pads of the chip must be accessible for external wire-bonding to a printed circuit board, PCB. The experimental platform we present in this paper satisfies all the above-mentioned requirements; in addition, its modular approach allows its implementation in a wide range of MEMS applications.

Figure 1 depicts an exploded rendering of the complete experimental platform. Its central component is a custom-made connector in PEEK material (see Fig. 2). We manufacture the connector with embedded channels that can be aligned to our chip microfluidic inlets. The fluidic path between the chip and the connector is hermetically sealed by ethylene propylene diene monomer (EPDM) rubber o-rings (Isoswiss, c.s. $0.50 \mathrm{~mm}$, i.d. $0.90 \mathrm{~mm}$ ). The connector is designed with a pair of fluidic channels in parallel connected to each SMR inlet. One channel is connected to an automated syringe pump (CETONI GmbH, neMESYS low-pressure module) with the help of polytetrafluoroethylene (PTFE) tubes (IDEX-HS, o.d. $1 / 16$ ", i.d. $0.02 ")$ and microfluidic fittings and ferrules (IDEX-HS, XP-206). The second channel is a by-pass channel and is either left open or closed by an external valve. This configuration allows us to quickly exchange the fluid flowing in the resonators, as we show later.

We design the PEEK connector with a central cavity, allowing for visual access to the resonators (both for microscope inspection and for laser-based readout of the motion of the resonators). This cavity also serves as an on-chip vacuum chamber: it is sealed towards the front-side of the chip by using nitrile o-ring (A. Aubry, c.s. $1 \mathrm{~mm}$, i.d. $8 \mathrm{~mm}$ ) that encloses the smaller microfluidic o-rings; it is sealed

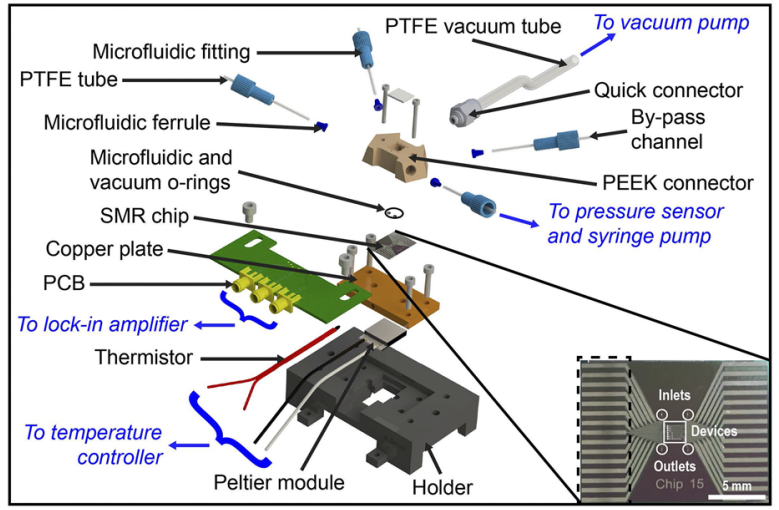

FIG. 1. Exploded rendering of the complete experimental platform and details of a typical SMR chip used for the experiments. The chip has electrode pads on the sides, a sensing window containing the resonators in the center, and fluidic inlets and outlets around. The 3D STEP file of the 3D printed holder is available elsewhere. [Associated dataset available at https://doi.org/10.5281/ zenodo.2605213]. . $^{14}$

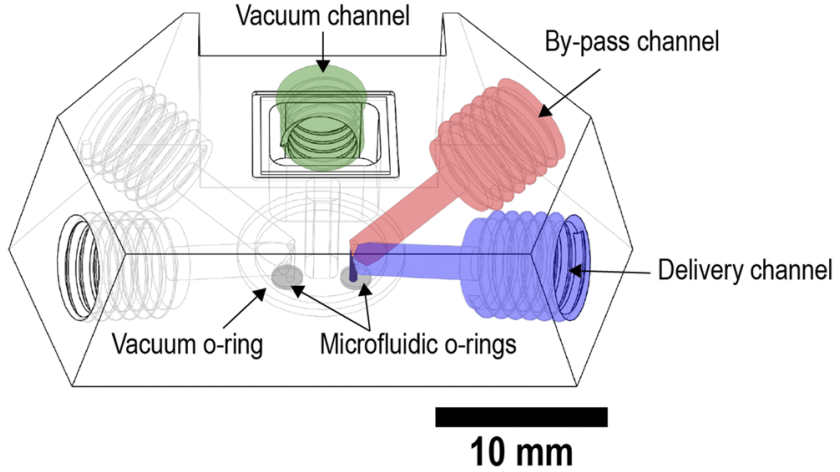

FIG. 2. Transparent schematic view of the manufactured PEEK connector, highlighting the purpose of by-pass channels. In purple: delivery channel connected to the pressure sensor and the syringe pump. In red: by-pass channel allowing to significantly lower the fluidic resistance of the platform when filling and exchanging fluids. In dark blue: remaining volume to be pushed through the SMRs during filling and exchanging fluids. In green: channel connected to the vacuum pump for operation in vacuum. The location of the vacuum and microfluidic o-rings is also shown. The drawing, along with the 3D STEP file of the connector, is available elsewhere. [Associated dataset available at https://doi.org/10.5281/zenodo.2605213].

on the top by using a glass slide with epoxy glue (3M, DP100 Clear). The vacuum in our cavity chamber is performed by connecting it to a turbo pump (Pfeiffer, HiCube) using a PTFE tube attached with quick connectors (Legris, 310106 19).

Temperature control is achieved by placing a custom-made copper plate underneath the chip while a droplet of thermal paste (Wakefield solutions) ensures good thermal conductivity. A thermistor (US Sensor, PR103J2) is inserted in the copper plate, in a cavity filled with thermal paste, and detects the temperature inside the plate. A Peltier module (Marlow Industries, RC3-4-01LS) is put in direct contact underneath the plate. These two components are connected to a thermoelectric temperature controller (Newport, ILX Lightwave LDT-5910C TTC), which builds a proportional integral derivative (PID) feedback loop to maintain constant the temperature (with a stability below $5 \mathrm{mK}$ over $5 \mathrm{~min}$ ) detected by the thermistor.

Finally, a PCB is attached in close contact to the chip and the electrode tracks are wire-bonded for electrical transduction of the devices.

Pictures of the assembled setup can be found in the supplementary material.

\section{RESULTS}

In this section, we demonstrate the functionality of our platform on three particular points: quick exchange of fluids, short atmospheric to vacuum pump-down time, and control of the temperature.

\section{A. Exchange of fluids with by-pass channels}

In the fluidic connector, the implementation of two channels in parallel connected to the chip inlet allows quick exchange of fluids in the devices. We first connect one channel to the syringe pump, 


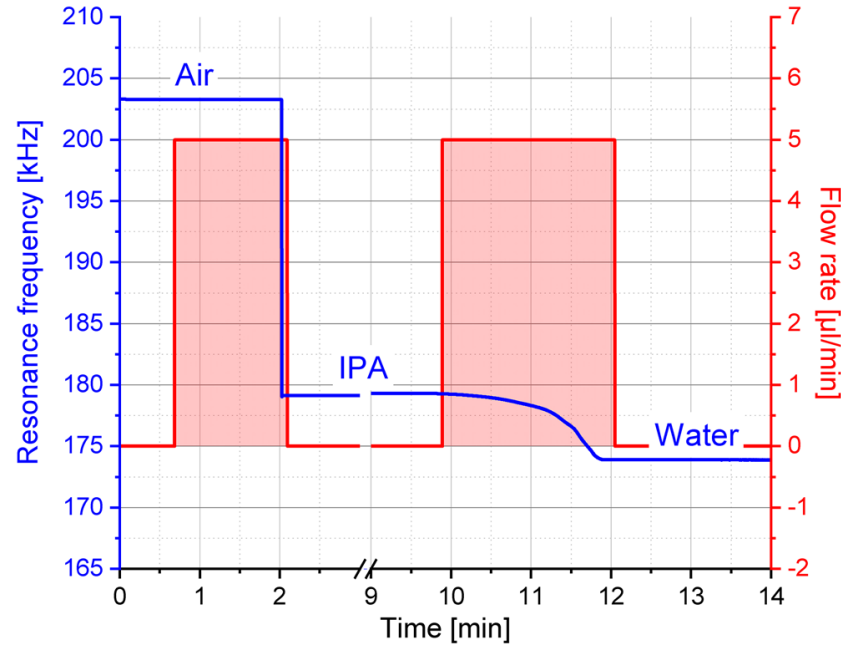

FIG. 3. Fast exchange of fluids demonstrated in a $250-\mu \mathrm{m}$-long SMR. A flow rate of $0 \mu \mathrm{l} / \mathrm{min}$ corresponds to manual operations (changing the syringe, flushing through the by-pass, closing the valve), while a flow rate of $5 \mu \mathrm{l} / \mathrm{min}$ is applied to exchange fluids in the SMRs. It takes only $85 \mathrm{~s}$ to replace air by IPA and $130 \mathrm{~s}$ to replace IPA by water.

while the other is left open. As the liquid is pushed from the pump, the enormous difference in fluidic resistance between the SMR channels (microscale diameter) and the by-pass channel (millimeter-scale diameter) makes the whole fluid go through the open by-pass, filling the red volume in Fig. 2. Once the whole fluidic line is filled, we close the by-pass channel, so that the remaining volume to be pushed through the SMRs consists only of the dark blue volume in Fig. 2. This configuration reduces the required time to exchange fluids in the resonator from several hours to few minutes, as shown in Fig. 3. We measure that exchanging air with isopropyl alcohol (IPA) takes around $85 \mathrm{~s}$, while replacing IPA with water takes around $130 \mathrm{~s}$. For the experiments shown in Fig. 3, a zero flow-rate on the graph corresponds to manual operations, such as opening/closing the bypass channel, exchanging the fluidic syringe, or filling the fluidic line. When fluids are pushed through the SMRs, a flow-rate of $5 \mu \mathrm{l} / \mathrm{min}$ is applied. A pressure sensor is installed between the syringe pump and the delivery channel to monitor the pressure in the SMRs. A pressure of 10 bars was maintained in the fluidic line for several hours and no damage to the devices nor leakages were observed.

\section{B. Vacuum-on-chip}

As previously mentioned, the experimental setup enables invacuum operation of our devices. The vacuum line from the connector to the pump includes a pressure gauge (Pfeiffer, PKR251). Nevertheless, that point is out of our custom-made vacuum chamber, which is too small to hold a gauge. Indeed, the pressure sensor is connected to the chamber via a PTFE tube of small inside diameter $(4 \mathrm{~mm})$ that induces a high fluidic resistance. Therefore, the pressure measured by the gauge does not accurately reflect the pressure in the chamber.

In order to assess the vacuum level in the custom-made chamber, we proceed with an indirect measurement of the pressure, using the quality factor of our SMRs as pressure sensors. It is well known

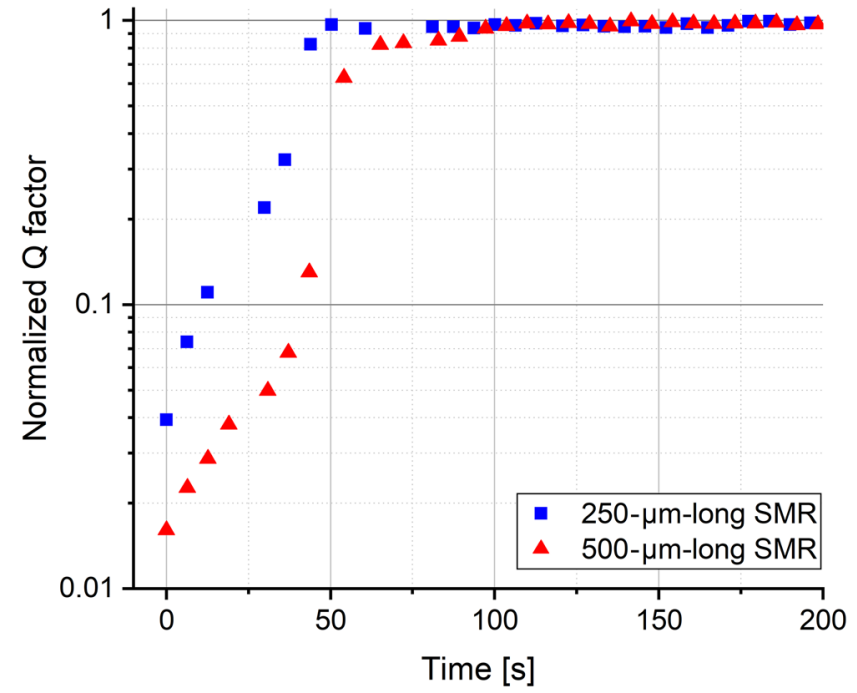

FIG. 4. Time needed to pump our custom-made vacuum chamber down. We notice that intrinsic quality factors are reached with $250-\mu \mathrm{m}$ - and $500-\mu \mathrm{m}$-long SMRs within 2 min of pumping. It is a tremendous improvement over the use of the commercial vacuum chamber, where a comparable level of vacuum is reached in around $3 \mathrm{~h}$.

that the quality factor of resonators depends on the pressure and that it saturates at low pressures. ${ }^{6,15} \mathrm{We}$ first measure the intrinsic quality factors of $250-\mu \mathrm{m}$ - and 500- $\mu \mathrm{m}$-long SMRs at low pressure (below $10^{-3}$ mbar $)$ in a big commercial vacuum chamber $\left(\sim 5000 \mathrm{~cm}^{3}\right.$ volume, $\sim 1850 \mathrm{~cm}^{2}$ surface area) where the pressure readings of the gauge are accurate (see supplementary material). We then assemble the characterized chip with our custom-made vacuum chamber $\left(\sim 0.2 \mathrm{~cm}^{3}\right.$ volume, $\sim 3 \mathrm{~cm}^{2}$ surface area) and start the vacuum pump. Figure 4 shows that intrinsic quality factors are reached in less than 2 min of pumping. As a comparison, it takes around $3 \mathrm{~h}$ to achieve the same results with the commercial chamber (much larger in volume and surface area). During experiments, the pressure gauge measures pressures as low as $10^{-5}$ mbar.

\section{Temperature control}

The resonance frequency of M/NEMS devices correlates with their temperature. ${ }^{16}$ In order not to be affected by temperature fluctuations of the chip and thus make accurate measurements of fluid samples flowing in the resonators, it is important to keep the temperature as stable as possible.

As we have mentioned before, the PID controller we utilize allows for a temperature stability of the thermistor below $5 \mathrm{mK}$ (over $5 \mathrm{~min}$ ). We can verify this by monitoring the resistance of the thermistor over time as a direct measure of the temperature. Naturally, this does not reflect the temperature of the SMR. Showing temperature stability of the SMR is actually rather difficult, because there is no magnitude that can be easily used for that purpose.

Instead, we show that our setup is able to control and stabilize the temperature of the chip at any set point between $25^{\circ} \mathrm{C}$ and $50{ }^{\circ} \mathrm{C}$ (the maximum temperature we successfully and repeatedly reach is $55^{\circ} \mathrm{C}$ ). To do that, we measure the resonance frequency of SMR devices in a vacuum environment, with fluidic channels 
empty (filled with air) and filled with deionized (DI) water, as a function of temperature and we compare to an analytical model to show excellent matching with the experimental results.

The temperature responsivity of the resonance frequency of a cantilever SMR in vacuum is expressed by

$$
\frac{1}{f} \frac{\partial f(T)}{\partial T}=\frac{1}{2} \beta_{\mathrm{SiNx}}+\frac{1}{2} \alpha_{\mathrm{SiNx}}-\frac{1}{1+\gamma_{\frac{\rho_{s}}{\rho_{f}}}}\left(\frac{3}{2} \alpha_{\mathrm{SiNx}}+\frac{1}{2} \frac{1}{\rho_{f}} \frac{\partial \rho_{f}(T)}{\partial T}\right),
$$

where $\beta_{\mathrm{SiNx}}=\frac{1}{E} \frac{\partial E(T)}{\partial T}$ is the temperature coefficient of the Young's modulus of the structural material (low-stress silicon nitride, ls- $\mathrm{SiN}_{\mathrm{x}}$ ) and $\alpha_{\mathrm{SiNx}}$ is the coefficient of thermal expansion of the solid. $\rho_{s}$ and $\rho_{f}$ are the densities of the solid and the fluid, respectively, and $\gamma=\frac{A_{s}}{A_{f}}$ is the ratio of the cross-sectional areas of the solid and the fluid.

When devices are empty (air inside the channels), the temperature responsivity is constant over the studied range of temperatures and has a negative sign, as can be seen in Fig. 5 (squares and solid lines). This is due to the softening of the ls- $\mathrm{SiN}_{\mathrm{x}}$, expressed by the term $\beta_{\mathrm{SiNx}}$ in Eq. (1), which is the dominating effect in this configuration, as has been reported before.

When the devices are filled with DI water, the variation of the density of the fluid with temperature, $\frac{1}{\rho_{f}} \frac{\partial \rho_{f}(T)}{\partial T}$, becomes the dominating term in Eq. (1): as the temperature increases, the density of water decreases, resulting in a responsivity with a positive sign, as shown in Fig. 5 (triangles and dashed lines). Table I summarizes the responsivities for empty and filled devices. The model for the filled devices was built taking the experimental values of the

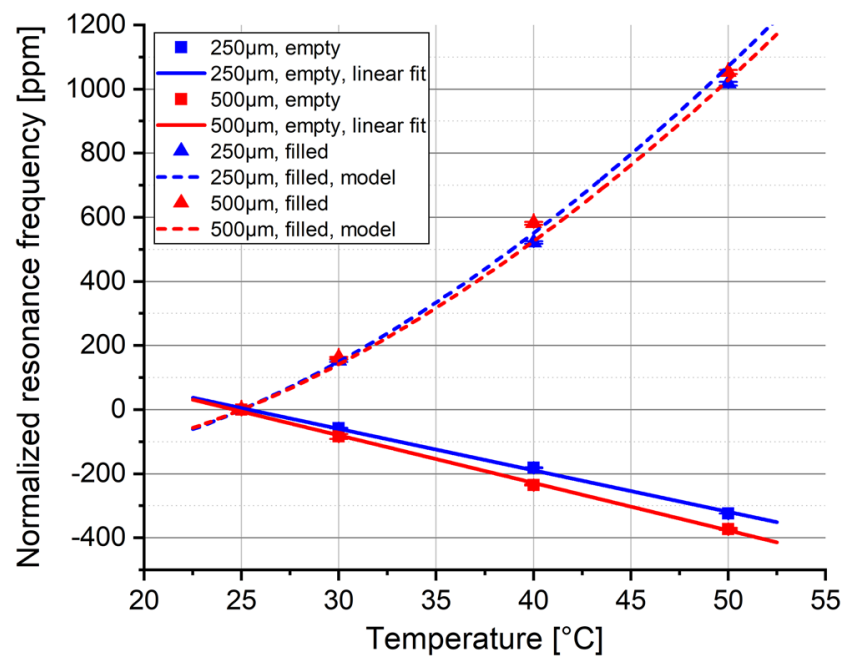

FIG. 5. Behavior of the resonance frequency of empty and filled $250-\mu \mathrm{m}$ - and 500- $\mu \mathrm{m}$-long SMRs with temperature. Empty data are represented by squares, while filled measurements correspond to triangles. The behavior of empty devices is well fitted by a linear function and thus shows a negative constant responsivity, as predicted by the theory. Using the responsivities of empty devices and the theoretical densities of water, we modeled the behavior of filled devices and observed good agreement with the data.
TABLE I. Fitted temperature responsivities of empty and filled devices. The measurements were taken with $250-\mu \mathrm{m}$ - and $500-\mu \mathrm{m}$-long SMRs, in a range of temperatures between $25^{\circ} \mathrm{C}$ and $50^{\circ} \mathrm{C}$.

\begin{tabular}{lll}
\hline \hline \multirow{2}{*}{ Device } & \multicolumn{2}{c}{ Fitted responsivities $\left(\mathrm{ppm} /{ }^{\circ} \mathrm{C}\right)$} \\
\cline { 2 - 3 } & \multicolumn{1}{c}{ Empty } & Filled (DI water) \\
\hline & & At $25^{\circ} \mathrm{C}: 26.34$ \\
& & At $30^{\circ} \mathrm{C}: 32.01$ \\
& $-12.94 \pm 0.09$ & At $40^{\circ} \mathrm{C}: 43.36$ \\
& At $50{ }^{\circ} \mathrm{C}: 54.70$ \\
\hline $500-\mu$ m-long SMR & At $25^{\circ} \mathrm{C}: 32.05$ \\
& $-14.84 \pm 0.12$ & At $30^{\circ} \mathrm{C}: 36.19$ \\
& At $40^{\circ} \mathrm{C}: 44.47$ \\
& At $50{ }^{\circ} \mathrm{C}: 52.76$ \\
\hline
\end{tabular}

responsivities for the empty devices, the theoretical densities of water, and the fitting parameter $\gamma$. In Fig. 5 , it is shown that the measured shifts in frequency agree well with the theory $(\gamma=0.7)$, indicating that our temperature control works adequately.

\section{CONCLUSION}

In this paper, we introduce a novel experimental platform for $\mathrm{M} / \mathrm{NEMS}$. It is devised and designed to satisfy the requirements for the characterization of suspended microchannel resonators and, as such, it combines fluidic delivery, fast exchange of fluids, vacuumon-chip, optical access to the devices, temperature control, and electrical connection. Nevertheless, the modular approach of the setup allows us to use it in different configurations.

In order to demonstrate the potential of our platform, we monitor the time needed for exchanging fluids in the device and found periods in the order of $100 \mathrm{~s}$. Additionally, we characterize the level of vacuum in the custom-made chamber, and we determine that intrinsic quality factors (pressure below $0.01 \mathrm{mbar}$ ) can be reached within less than $2 \mathrm{~min}$. Finally, we show good control of the temperature of the chip, and the temperature responsivity of the devices, both empty and filled, is measured in a range between $25{ }^{\circ} \mathrm{C}$ and $50{ }^{\circ} \mathrm{C}$, with the results matching closely the behavior predicted by the theory.

As a conclusion, we believe that the solution introduced in this paper can be useful not only for SMRs but also for the study of many different MEMS and NEMS devices.

\section{SUPPLEMENTARY MATERIAL}

See supplementary material for more details on the characterization of the vacuum on chip and the experimental setup.

\section{ACKNOWLEDGMENTS}

The authors acknowledge financial support from the Swiss SNF (Grant No. PP00P2_170590) and MCSA (MC-IEF-706312). They also thank the ATME mechanical workshop at EPFL for manufacturing the PEEK connector. 


\section{REFERENCES}

${ }^{1}$ R. R. A. Syms and D. F. Moore, Mater. Today 5, 26-35 (2002).

${ }^{2}$ A. C. R. Grayson, R. S. Shawgo, A. M. Johnson, N. T. Flynn, Y. Li, M. J. Cima, and R. Langer, Proc. IEEE 92, 6-21 (2004).

${ }^{3}$ J. Tamayo, P. M. Kosaka, J. J. Ruz, Á. San Paulo, and M. Calleja, Chem. Soc. Rev. 42(3), 1287-1311 (2013).

${ }^{4}$ R. Bogue, Sens. Rev. 33, 300-304 (2013).

${ }^{5}$ Y. Temiz, R. D. Lovchik, G. V. Kaigala, and E. Delamarche, Microelectron. Eng. 132, 156-175 (2015).

${ }^{6}$ S. Schmid, L. G. Villanueva, and M. L. Roukes, Fundamentals of Nanomechanical Resonators (Springer, 2016).

${ }^{7}$ C.-C. Yang and Y.-L. Hsu, Sensors 10, 7772-7788 (2010).

${ }^{8}$ T. P. Burg and S. R. Manalis, Appl. Phys. Lett. 83(13), 2698-2700 (2003).

${ }^{9}$ A. De Pastina, D. Maillard, and L. G. Villanueva, Microelectron. Eng. 192, 83-87 (2018).

${ }^{10}$ M. F. Khan, S. Schmid, P. E. Larsen, Z. J. Davis, W. Yan, E. H. Stenby, and A. Boisen, Sens. Actuators, B 185, 456-461 (2013).
${ }^{11}$ M. Godin, A. K. Bryan, T. P. Burg, K. Babcock, and S. R. Manalis, Appl. Phys, Lett. 91(12), 123121 (2007).

${ }^{12}$ T. P. Burg, M. Godin, S. M. Knudsen, W. Shen, G. Carlson, J. S. Foster, K. Babcock, and S. R. Manalis, Nature 446(7139), 1066-1069 (2007).

${ }^{13}$ N. Cermak, S. Olcum, F. F. Delgado, S. C. Wasserman, K. R. Payer, M. A. Murakami, S. M. Knudsen, R. J. Kimmerling, M. M. Stevens, Y. Kikuchi, A. Sandikci, M. Ogawa, V. Agache, F. Baléras, D. M. Weinstock, and S. R. Manalis, Nat. Biotechnol. 34(10), 1052-1059 (2016).

${ }^{14}$ D. Maillard, A. De Pastina, T. Larsen, and L. G. Villanueva (2019), "Modular interface and experimental setup for in-vacuum operation of microfluidic devices," Zenodo. https://doi.org/10.5281/zenodo.2605213.

${ }^{15}$ K. B. Gavan, J. van der Heijden, E. van der Drift, and H. van der Zant, in Proceedings of IEEE Sensors (IEEE, 2009), pp. 1836-1839.

${ }^{16}$ M. Sansa, E. Sage, E. C. Bullard, M. Gély, T. Alava, E. Colinet, A. K. Naik, L. G. Villanueva, L. Duraffourg, M. L. Roukes, G. Jourdan, and S. Hentz, Nat. Nanotechnol. 11(6), 552-558 (2016).

${ }^{17}$ T. Larsen, S. Schmid, S. Dohn, J. E. Sader, A. Boisen, and L. G. Villanueva, J. Micromech. Microeng. 27(3), 035006 (2017). 